\title{
Diacronie
}

Studi di Storia Contemporanea

$\mathrm{N}^{\circ} 15,3 \mid 2013$

Spazi, percorsi e memorie

\section{«A vencer o morir»: rivoluzione e militanza politica nell'Argentina degli anni Settanta}

La storia di Cacho Narzole rivoluzionario ed esule politico argentino

\section{Camillo Robertini}

\author{
(2) OpenEdition \\ Journals \\ Edizione digitale \\ URL: http://journals.openedition.org/diacronie/501 \\ DOI: $10.4000 /$ diacronie.501 \\ ISSN: 2038-0925 \\ Editore \\ Association culturelle Diacronie
}

Notizia bibliografica digitale

Camillo Robertini, « «A vencer o morir»: rivoluzione e militanza politica nell'Argentina degli anni

Settanta », Diacronie [Online], N 15, 3 | 2013, documento 8, Messo online il 01 octobre 2013,

consultato il 02 mai 2019. URL : http://journals.openedition.org/diacronie/501 ; DOI : 10.4000/ diacronie. 501 


\section{Diacronie}

\section{8/}

\section{«A vencer o morir»: rivoluzione e militanza politica nell'Argentina degli anni Settanta \\ La storia di Cacho Narzole rivoluzionario ed esule politico argentino}

A cura di Camillo ROBERTINI *

Il presente contributo offre una panoramica sulle vicende politiche, sociali e personali di un giovane rivoluzionario, Cacho Narzole, che attraverso il prisma della propria esperienza di militanza tratteggia una delle pagine più drammatiche della recente storia argentina. Impegnato nelle file dell'Ejército revolucionario del pueblo (ERP), formazione armata marxista-leninista attiva fra il 1970 e il 1977, descrive, nell'intervista che segue, particolarità e vicissitudini dell'esperienza della militanza armata: dal contesto sociale dell'Argentina degli anni Settanta alla traumatica esperienza dei desaparecidos, dall'esperienza dell'esilio forzato in Italia al ritorno alla democrazia. Una lunga intervista il cui obiettivo principale è quello di condensare in un unico spazio la decade iniziata con il ritorno di Juan Domingo Perón al potere e terminata con la sconfitta argentina alle isole Falkland/Malvinas (1973-1983).

\section{Militanza politica e militare in Argentina: un'introduzione}

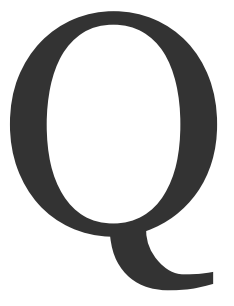

uesto studio si focalizza sulle esperienze di vita e di militanza politica di un componente del PRT-ERP (Partido Revolucionario de los Trabajadores - Ejercito Revolucionario del Pueblo), movimento nato in Argentina nel 1965, di tendenza marxista leninista, e liquidato durante la repressione del governo militare nel 1977. Le vicende dell'ERP, che saranno ripercorse parzialmente attraverso alcuni stralci di interviste registrate, sono da inserire in un complesso contesto politico che risente degli equilibri geopolitici, ma 
anche culturali, internazionali. Anche alcune caratteristiche peculiari della storia argentina vanno a costituire il substrato delle vicende e dei posizionamenti dell'Ejercito Revolucionario. La breve analisi proposta vuole fornire un inquadramento contestuale di tali stimoli interni ed esterni al paese, per poi soffermarsi sulle specificità della storia raccontata, attraverso le parole di militanti che svelano la loro individualità esperienziale e i loro processi di agency ${ }^{1}$.

Occuparsi della recente storia Argentina, specie per gli anni Settanta, impone di parlare del tema, storiograficamente molto dibattuto, della militanza politica.

Il contesto internazionale degli anni a cavallo fra Sessanta e Settanta è quello del continente latinoamericano contraddistinto da una serie di tensioni, che spesso sfociano in processi rivoluzionari. Non è solo il contesto latinoamericano a determinare la nascente tensione verso un "sentimento" rivoluzionario; nell'immaginario collettivo argentino la Guerra di Algeria e l'indipendenza conquistata dal FLN (Front de Libération Nationale) nel 1962 occupano un posto importante ${ }^{2}$. Gli ideali di giustizia e socialismo ottengono una grande eco internazionale, anche grazie a opere cinematografiche come La battaglia di Algeri di Gillo Pontecorvo3 (1962). Poco prima dell'indipendenza algerina, a Cuba (1959) si insedia un governo rivoluzionariocomunista: Che Guevara e Fidel Castro conquistano il potere grazie al sostegno popolare e dopo la loro vittoria cominciano a teorizzare metodi e forme del «buon rivoluzionario»4. Dalle teorizzazioni di Guevara si sviluppa una linea, che dà il via ad una particolare pubblicistica, quella rivoluzionaria, che culminerà con l'opera globalmente più conosciuta e riconosciuta dai rivoluzionari e dai giovani di un'intera generazione: il libretto rosso di Mao Tse-Tung (1966).

Il problema della guerriglia rivoluzionaria fu percepito in Argentina non tanto, come nel caso di Cuba o del Cile, come una necessità che si risolveva nella stessa immagine oleografica della rivoluzione, ma come unica possibile alternativa di partecipazione alla vita politica nazionale.

La Revolución libertadora del 1955 aveva di fatto bloccato la principale struttura politica nazionale: il Partito peronista. Un gruppo di ufficiali dell'esercito, appoggiati dai settori più conservatori dell'alta borghesia e della Chiesa cattolica, aveva deposto il governo di Juan Domingo Perón, poiché la sua spiccata sensibilità verso le classi

\footnotetext{
${ }^{1}$ TAYLOR, Charles, Introduction e What Is Human Agency?, in TAYLOR Charles (ed.) Human Agency and Language,Cambridge, Cambridge University Press, pp. 1-14.

${ }^{2}$ CAVIASCA, Guillermo Martìn, «La cuestión militar y las organizaciones guerrilleras argentinas» in Cuadernos de Marte,1, 2/2011, pp. 9-42.

${ }^{3}$ PONTECORVO, Gillo, La battaglia di Algeri, Igor Film-Casbah Film, Algeria-Italia, 1966, 121'.

${ }^{4}$ ZANATTA, Loris, Storia dell'America Latina contemporanea, Laterza, Roma-Bari, 2010.
} 
popolari - e la tendenza «totalitaria» ${ }^{5}$ del suo governo democratico - avevano determinato un allontanamento della borghesia dal Presidente. Nella vita politica argentina esistevano altri due partiti: quello Comunista, avulso dall'essere un organo di massa e serrato nella sua struttura interna, e quello Radicale (Unión Cívica Radical) espressione di un liberalismo oscillante fra la destra sociale e il laissez-faire economico $^{6}$. L'assenza di alternative, quindi, costrinse progressivamente una generazione di argentini a scegliere la militanza rivoluzionaria quale forma di "evasione" da una "gabbia" partitica che sembrava impenetrabile".

Come se non fosse bastato, anche la situazione internazionale continuò a declinare verso uno scenario politico incerto: nel 1973 Pinochet fu al vertice di un golpe che destituì il socialista Allende dalla presidenza cilena, inaugurando nel Cono Sur una dittatura militare particolarmente efferata. Questi avvenimenti, che scuotono tutto il continente e si caratterizzano per violenza e processi repressivi, creano un'eco che influisce quindi anche in Argentina nella creazione di una coscienza rivoluzionaria condivisa.

Parallelamente altri fattori internazionali giocarono un ruolo di rilievo nello svilupparsi della guerriglia. Il Maggio francese (1968) aveva spianato la strada - su di un piano intellettuale - alla prospettiva rivoluzionaria e le relazioni con gli ambienti accademici e intellettuali europei, e con la stessa produzione politologica e criticomarxista della Scuola di Francoforte, trovarono un posto di primo piano nella formazione di militanti spiccatamente rivoluzionari. Ad aggiungersi al quadro, è da sottolineare 1'apporto ideologico delle forme di guerriglia organizzata che già si erano sviluppate in America Latina negli stessi anni: ad esempio le Fuerzas Armadas Para la Liberación Nacional (FALN) in Venezuela ${ }^{8}$ che avevano ispirato dalla fine degli anni Sessanta il sorgere di movimenti molto simili in paesi come Colombia e Cuba.

Ritornando alla politica interna del Paese, il peronismo, seppur virtualmente assente dal 1955 con l'esilio del suo leader, aveva promosso e foraggiato il sorgere di movimenti armati al suo interno, attraverso una serie di strutture organizzate

\footnotetext{
${ }^{5}$ BENEDINI Giuseppe, Il peronismo, la democrazia totalitaria in Argentina, Roma, Editori Riuniti, 2011.

${ }^{6}$ ROMERO Luis Alberto, Breve historia contemporánea de la Argentina, 1916-2010, Buenos Aires, Editorial Santa Fe, 2011, p. 100.

${ }^{7}$ SALCEDO Javier, Los Montoneros del barrio, Caseros, EDUNTREF, 2011, pp. 32-35.

${ }^{8}$ LIMÓN Ricardo, «El Partido Comunista de Venezuela: Sus tácticas políticas de 1964 a 1969», in Foro Internacional, 11, 4/1971.
} 
clandestinamente $^{9}$. Il più rappresentativo di questi gruppi fu quello dei Montoneros, il cui nome deriva dai caudillos che governavano i distretti federali dell'Argentina poco dopo l'indipendenza, legati alla figura di José de San Martín, generale e combattente delle Province Unite del Río della Plata (l'embrione della Repubblica Argentina). Montoneros si contraddistinse per essere un soggetto radicato in ogni singolo barrio (quartiere) delle grandi città argentine (Buenos Aires, Córdoba, Rosario) che lottava, attraverso una strategia di sequestri e assalti a centri di potere, per la vuelta (ritorno) di Perón al potere e per la «patria socialista».

Al di fuori della culla peronista, tuttavia, si svilupparono altri esperimenti tesi al ribaltamento dell'ordine costituito attraverso un processo rivoluzionario. Il principale esercito rivoluzionario di sinistra fu il Partido de los Trabajadores Argentinos-Ejercito Revolucionario del Pueblo (PRT-ERP) che nacque nel 1969 e si confrontò con le contestualità internazionali sopra descritte, ma anche con una serie di gruppi, rivoluzionari, ma non guerriglieri, che avevano animato la vita politica argentina ${ }^{10}$. Era questo il caso del Movimiento Peronista de Liberación-Ejercito de Liberación Nacional (MPL-ELN) che fu attivo specialmente nella provincia rurale di Tucumán, il quale si sviluppò contestualmente alla Rivoluzione cubana senza riuscire a "importarla" in Argentina. Nel caso del MPL-ELN, ma soprattutto in quello di Montoneros, che come sopra accennato era il vero e proprio «braccio armato del peronismo» ${ }^{11}$, Perón continuò a rivestire un ruolo di primo piano nel controllo e nella gestione degli stessi. Il generale, dal suo esilio in Spagna, esercitò un controllo quasi incondizionato su tre strutture politiche fondamentali dell'Argentina: il sindacalismo di Stato della CGT (Confederacion Genral del Trabajo) il movimento rivoluzionario Montoneros e il proscritto Partito justicialista.

È fuori da ogni dubbio che l'apparato politico di Perón, prendendo dal populismo latinoamericano una forte carica personalistica e dal fascismo sociale una certa propensione verso le classi subalterne, fu nella pratica un organismo politico "difforme" con anime estremamente antitetiche al proprio interno, capaci, tuttavia, di rinunciare alla propria individualità di fronte al proprio capo. A questo va aggiunto che le dichiarazioni

\footnotetext{
${ }^{9}$ TORRE, Juan Carlos (dir. por), Nueva Historia Argentina, t. VIII, Los años peronistas (1943-1955), Buenos Aires, Sudamericana Editorial, 2000.

${ }^{10}$ MATTINI, Luis, Hombres y mujeres del PRT-ERP, De la Campana, La Plata, 1988.

${ }^{11}$ DE SANTIS, Daniel, La historia del PRT-ERP por sus protagonistas, Buenos Aires, Editorial Guevarista, 2010, pp. 189-201.
} 
pubbliche di Perón, cercarono sempre di interpretare i sentimenti vissuti dagli argentini nella maniera più viscerale, riuscendo a tenere assieme, in un difficile gioco di equilibrismo, le diversissime anime del proprio movimento. La lettera inviata ai propri compagni di partito in occasione della morte di Che Guevara in Bolivia manifesta proprio questa tensione:

\begin{abstract}
Oggi è caduto durante la lotta, come un eroe, la figura più straordinaria della rivoluzione latinoamericana: è morto il Comandante Ernesto "Che" Guevara. La sua morte mi dilania l'anima perché era uno dei nostri, chissà il migliore [...] La profonda convinzione della giustezza della causa che abbracciò, gli dette la forza, il valore, e il coraggio che oggi lo eleva alla categoria di eroe e martire ${ }^{12}$.
\end{abstract}

Sono parole, espresse a caldo da Perón, che esprimono a pieno titolo speranze e sensazioni condivise quasi totalmente da una generazione di Latinoamericani. Queste affermazioni persuadono i peronisti radicali del fatto che il movimento assumerà, tornato in patria il pripro conductor (comandante), un programma rivoluzionario. Parole come queste vanno a costituire una vera e propria «legittimazione sociale» ${ }^{13}$ come 1 'ha definita il sociologo Gullermo O'Donnel, che di fatto preparano il terreno per un decennio che si caratterizza per il dominio della violenza politica e al contempo per il sorgere di movimenti rivoluzionari di quasi tutte le tendenze politiche. In Argentina poi si passerà dalla semplice legittimazione della lotta armata all'appoggio diretto alla stessa da parte di alcuni settori (studenti, operai, classi popolari).

Questi elementi sembrerebbero sufficientemente coerenti per comprendere come dal 1969, data simbolo della storia argentina, si sviluppi una storia di lotta armata che culmina con il golpe del generale Videla il 24 marzo 1976. Nel 1969 a Córdoba si era sperimentata un'esplosiva alleanza fra studenti e operai che, partendo da una rivendicazione salariale, si era sviluppata come una vera e propria sommossa (pueblada). Il Cordobazo, come è definita la sommossa cittadina, oltre a mettere in crisi il regime dei militari di Onganía, che di lì a poco si vedrà costretto a cedere il potere a

\footnotetext{
${ }^{12}$ PERÓN, Juan Domingo, cit. in, Norberto GALASSO, Perón: Exilio, resistencia, retorno y muerte, 1955-1974, Buenos Aires, Colihue, 2005, p. 1011.

${ }^{13}$ O’DONNELL, Guillermo, « ¿Y a mi, que me emporta? Notas sobre sociedad y politica en Argentina y Brazil», Working paper 9, January 1984, URL:

< http://kellogg.nd.edu/publications/workingpapers/WPS/009.pdf > [consultato il 3 ottobre 2013]. 
Lanusse e successivamente a ripristinare l'iter democratico (1973), dà il via a una ola (onda) di scontri e proteste ${ }^{14}$. ̀̀ in questo frangente che inizia la storia di George, che fin dal 1969 prende il nome di battaglia di Cacho. La storia di Cacho è quella di una generazione: una storia spezzata e terminata per molti con la sparizione e con la tortura e per altri, come nel caso dello stesso Chacho con l'esilio, unica via di scampo da una dittatura liberale e "normale" di giorno, violenta e genocida di notte.

\section{L'intervista a Cacho Narzole}

L’intervista a Cacho Narzole, guerrigliero ed esule politico argentino, condotta a Buenos Aires all'interno di un programma di ricerca sulla militanza politica nell'Argentina degli anni Settanta è l'espediente per porre alcune questioni sul tema. Il protagonista di questa storia pone l'accento su alcuni temi connessi all'essere rivoluzionario nel contesto dell'America Latina: dall'influenza dell'intellettualità europea nella formazione di una "coscienza rivoluzionaria" ai rapporti di classe nell'Argentina degli anni Settanta. Ma più genericamente la questione che in queste pagine si esamina è l'evoluzione della società di fronte all'ultima dittatura militare (1976-83).

Camillo Robertini: Studiare la storia Argentina negli anni della dittatura, porta irrimediabilmente a parlare degli anni precedenti, che rappresentarono per la vostra generazione, la generazione del Settanta, un momento di effervescenza intellettuale e di utopia rivoluzionaria irripetibile. Per lei che militò nella sinistra rivoluzionaria che senso assunse la lotta?

Cacho Narzole: A partire dagli anni Settanta, quello che provoca il primo movimento dentro la gioventù argentina è la rivoluzione cubana, che parte quando i rivoluzionari prendono il potere a Cuba... qui ha un effetto fortissimo e tutti i giovani incominciano a chiedersi «perché non è possibile fare lo stesso?» e allora incominciano le riunioni, si parla di quello che si può fare e nascono moltissime attività politiche, al di fuori di quello che erano le strutture politiche classiche, come i partiti politici dell'Argentina.

\footnotetext{
${ }^{14}$ ADAMOVSKY Ezequiel, Historia de las clases populares en la Argentina, Buenos Aires, Sudamericana Editorial, 2012, p. 168.
} 
Principalmente fuori dalla struttura della sinistra, ovvero il Partito comunista ${ }^{15} \ldots$ si comincia con lo studio dell'esperienza cubana attraverso i libri del Che e di tutto il discorso di Fidel... e dopo incomincia ad apparire il grande movimento che si stava sviluppando in Cile... un movimento che già prima di Allende, che arrivò dopo quattro $o$ cinque anni, all'inizio degli anni Sessanta già si faceva strada... era l’idea che si potesse installare un'alternativa. L'altro tema centrale per la nostra generazione ha a che fare con la guerra del Vietnam, che fu qualcosa che per noi aprì la possibilità reale che gli USA, con tutta la loro potenza, non potessero contrastare la rivoluzione, che era combattuta da un piccolo popolo.

$\mathrm{Si}$ incominciò a discutere di questo, anche quando incominciò a nascere una bibliografia su questo tema, i libri di Hồ Chí Minh, il Libretto rosso di Mao...

In America Latina semplicemente l'esperienza dei Tupamaros in Uruguay, che fu l'avanguardia... ebbe una grande ripercussione sul movimento, anche perché si parlava molto di loro, e poiché incominciammo a studiare anche questa esperienza.

Molte cose succedevano anche in Europa: il Maggio francese aprì una fase di discussione politica enorme. Ma non si limitava alla discussione, si stava sviluppando una effervescenza editoriale, il cinema parlava degli stessi problemi... il film $L a$ battaglia di Algeri per esempio... sono tutte cose che produssero un forte impatto sui movimenti giovanili che erano realmente commossi, partecipi... avevamo una voglia fortissima di impegno e di azione, ma ancora non sapevamo come canalizzarla in un luogo preciso, che pensavamo sarebbe dovuto essere un nuovo soggetto rivoluzionario argentino... per costruirlo andammo dove era presente una maggiore conflittualità sociale. In Argentina nella provincia di Tucumán ${ }^{16}$ vi era molta mobilitazione anche perché il programma politico degli Stati Uniti17, aveva imposto un determinato sviluppo economico continentale che escludeva dal benessere una grande quantità di persone. Si assisteva quindi alla chiusura delle fabbriche, all'ampliarsi dell'ineguaglianza... tutte cose che un settore giovane della popolazione osservava e per questo andava sul posto.

\footnotetext{
${ }^{15}$ Il Partito comunista argentino, fu fondato nel 1918 dopo una scissione dal Partito socialista argentino. La storia del partito segue quella delle dittature civico-militari dell'Argentina per arrivare all'opposizione col peronismo. Negli anni Settanta pur mantenendo un certo influsso sui giovani rivoluzionari, si inserì nella scia dei partiti politici tradizionali argentini. Per un approccio preliminare Cfr. ZANATTA, Loris, Il peronismo, Roma, Carrocci, 2008.

${ }^{16}$ La provincia di Tucuman situata nel nord-ovest dell'Argentina è tradizionalmente una regione contadina caratterizzata dalla presenza della canna da zucchero. Negli anni Settanta si sviluppò uno scontro per la proprietà della terra. Si formarono delle leghe contadine capeggiate da sindacalisti locali e di Buenos Aires con lo scopo di ottenere le terre per i contadini. Successivamente l'ERP (Ejército revolucionario del pueblo) installerà lì il proprio principale fronte di combattimento. I militari dal 1974 assumeranno la direzione della provincia e da li partirà la repressione e i primi desaparesidos.

${ }^{17}$ L'intervistato fa riferimento alla Dottrina di sicurezza nazionale degli USA, che sostanzialmente sostenne finanziariamente e militarmente i regimi militari in America Latina.
} 


\section{R.: In questo senso possiamo parlare di una separazione all'interno} dell'Argentina fra una provincia dove la lotta si stava sviluppando e Buenos Aires e gli altri centri dove si viveva tutt'altro tipo di situazioni?

C. N.: Certamente c'erano enormi differenze fra quelle che erano le grandi concentrazioni urbane, come Buenos Aires, Cordoba, Rosario, e quello che era la provincia all'interno della quale si produce una convergenza... una sorta di combinazione esplosiva fra i settori intellettuali, soprattutto le università, che incominciarono a prendere contatto con gli operai. Si stava sviluppando un'avanzata della destra alla quale risposero gli studenti. In quel momento incominciarono a impedire e ostacolare le attività politiche nelle università, si approvarono delle leggi di limitazione dell'attività giovanile... tutto questo però generò una specie di resistenza, che durò quattro o cinque anni... avevamo molta voglia di fare, anche se non sapevamo come dirigere le nostre forze.

Da questo inasprimento iniziale in molti incominciammo a incamminarci verso la lotta armata, che a partire dall'esperienza che si viveva in tutto il mondo, specie per la decolonizzazione dell'Africa e la lotta d'indipendenza algerina e angolana, sembrava una via possibile. Non possiamo dimenticare neanche l'effervescenza intellettuale europea... tutto quello che producevano gli intellettuali francesi, italiani, generava permanentemente un dibattito politico e un grande avanzamento della sinistra argentina. Un'avanzata che era del tutto simile a quella dei partiti comunisti europei negli anni Settanta... un'ondata che sembrava, in tutto il mondo, non si potesse fermare... avevamo l'impressione che tutto il mondo andasse nella stessa direzione. Per noi il principale problema era che i partiti politici argentini non erano dei contenitori capaci di generare risposte, o nei quali poter aprire un dialogo.

C. R.: Provaste ad interloquire con il Partito peronista, il principale movimento politico argentino, che manteneva anche in questi anni una carica rivoluzionaria?

C. N.: Il peronismo era una struttura politica che, possiamo dire, aveva goduto sempre di una posizione di resistenza, in quanto era stato proibito come partito e viveva nella 
clandestinità, ma che sempre aveva mantenuto una struttura politica fortemente verticale e poco democratica, perché al suo interno tutto era determinato dall'orientamento di Perón.

Il peronismo però, nonostante questo, era a favore del rispetto di un ordinamento democratico, del recupero di una legalità che in questo senso esigeva la partecipazione di tutti i partiti alla vita democratica, ma alcune parti dell'oligarchia argentina non intendevano concedere questo sviluppo della legalità. Gli anni Settanta spiegano anche quello che è il movimento peronista: un movimento di massa che nonostante tutto aveva una certa partecipazione giovanile, parlo della gioventù peronista, che sentì anche la necessità di ricorrere alla lotta $\operatorname{armata}^{18}$. Tutto questo anche perché negli Stati Uniti si era definita la strategia della sicurezza nazionale di Kissinger, che fondamentalmente era il contrasto ai movimenti comunisti in tutto il mondo.

In un mondo bipolare i settori della destra reazionaria consideravano prioritario l'allineamento con gli USA, mentre noi altri guardavamo al Blocco sovietico.

La necessità di questo allineamento reazionario in tutta l'America Latina, fra Sessanta e Settanta farà dei militari un vero e proprio partito politico, dotato della sua forza e di una linea precisa di azione. L'idea era di imporre una disciplina per riportare l'ordine nel paese in vista di uno preciso piano di sviluppo economico.

\section{R.: In questa descrizione rientra quindi anche il progetto della giunta militare argentina, quello della Reorganización Nacional ${ }^{19}$ del 1976?}

C. N.: Certamente il 1976 è il momento di maggiore gravità di questo processo di rigetto, che dal 1972-1974 si era sviluppato in senso reazionario. La cupola militare si stava diffondendo, perché nessun partito politico della destra aveva la forza politica per contrastare lo sviluppo della sinistra.

\footnotetext{
${ }^{18}$ L'intervistato fa riferimento al movimento rivoluzionario marxista-peronista Montoneros, che proprio nel congiunto fra le due ideologie traeva la sua ispirazione. Dedito alla guerriglia urbana e agli attentati rappresentò la sinistra estrema del peronismo. Il movimento fu stroncato nel 1976 dalla giunta militare. Per un primo approccio alla militanza rivoluzionaria peronista Cfr. MASSARI, Roberto, Il peronismo, Roma, Erre Emme, 1997. Per uno studio sistematico sull'azione di Montoneros Cfr. SALCEDO Javier, Los Montoneros del barrio, Caseros, EDUNTREF, 2011.

19 Propagandato dalla giunta militare argentina come un profondo programma di rinnovamento dell'architettura economica del paese, nella sostanza si inserisce nelle politiche di apertura neoliberali al mercato. Gli effetti del Processo, che già nel 1978 furono del tutto opposti a quelli preventivati, andarono nella direzione di minare la produzione industriale del paese e, al contempo, di abbattere lo stato sociale peronista, costruito nella seconda metà degli anni Cinquanta da Juan Domingo Perón.
} 


\section{R.: Però molti storici argentini hanno affermato che il golpe del 1976 fu tutto sommato ben accettato dalla popolazione argentina, questo non è in contrasto con quello che abbiamo detto?}

C. N.: Non è facile rispondere... quello che successe nella seconda metà degli anni Settanta fu che i settori della classe media argentina, incominciarono ad intravedere una certa convenienza nel programma economico che i militari proponevano. Perché questo si fondava principalmente sull'esclusione dei settori popolari dall'accesso al benessere economico, che, invece, volevano preservare per loro. In questo senso la classe medio era interessato, ma ovviamente non quella intellettuale... molto meno quella dell'avanguardia e quella degli operai e dei contadini che erano naturalmente i maggiori esclusi. Questo si può leggere chiaramente nelle parole del ministro dell'economia della prima giunta militare Videla, José Alfredo Martínez de Hoz, che dichiarò nel suo discorso iniziale che il paese poteva pensare a quindici milioni di persone, quando il paese ne aveva trenta: era evidente che la grande maggioranza del popolo sarebbe dovuta andar via o accettare le nuove condizioni di vita.

\section{R: Più che di un'accettazione possiamo quindi parlare di una mancanza di organizzazione delle classi popolari davanti al golpe e di connivenze nella classe media? Fu solo questo?}

Sì, le classi popolari per la loro vastità non erano facili da organizzare ed in un secondo momento la brutalità della repressione decapitò tutti i movimenti politici e sociali, al punto che non si poté organizzare alcuna resistenza. Ricordiamo che trentamila desaparecidos sono moltissimi... violenze, torture, sparizioni in questa quantità impressionarono tutti. In più si disarticolò tutta la struttura sociale argentina, attraverso il diffondersi dell'intimidazione e della paura... e questo processo repressivo ovviamente aveva trovato un valido sostegno nei mezzi di comunicazione, che furono occupati dai militari. In questo modo i mezzi di comunicazione proponevano una situazione politica che non rispondeva al vero, ma che era difficile smentire, non 
avevamo accesso ai mezzi di comunicazione per smentire, ma, se anche si fosse fatto, in poco tempo saremmo scomparsi.

\section{R.: Tutti i giornali furono occupati? Quindi «La Nacion» e il «Clarín» per parlare dei principali o fu un'operazione più vasta?}

C. N.: Certo, ma anche giornali popolari come «El Mundo», che fu bombardato ed assaltato... perché questo succedeva, quando c'era una voce che usciva dal coro: i militari facevano sparire fisicamente il direttore o il giornalista, oppure sequestravano tutto. Un caso particolare è quello del giornale «El Cronista Comercial»: pur essendo un giornale di ispirazione economica, che non aveva alcuna tendenza politica, davanti alla escalation degli anni 1973-1976 incominciò a parlare della violenza e delle cose che non andavano. La negazione delle libertà era davvero qualcosa che non accettavano. Il suo direttore Perrotta fu sequestrato dai militari, mentre veniva rimpiazzato con un giornalista di tendenze governative, e alla fine sparì... In questo senso la repressione si era fatta talmente forte che non colpi più quelli che erano i settori che "naturalmente" avrebbe dovuto reprimere, quelli della sinistra, ma anche chi, pur essendo di destra, assumeva una posizione critica nei confronti del regime.

In questo senso vivere in una società che veniva schiacciata dalla repressione non era facile, anche perché il timore era molto diffuso, al punto che si disintegrò quella rete sociale fondamentale per gli uomini.

C. R.: Di fronte a questa «atomizzazione della società» ${ }^{20}$, voi come opposizione e come movimento giovanile in che rapporto eravate con il sindacalismo e con la CGT? Le faccio questa domanda perché nel $1973 \mathrm{fu}$ approvata la legge per la regolamentazione del lavoro, che dette a molti l'impressione di un trionfo del proletariato e alla CGT un nuovo peso politico.

C. N.: Come in tutte le fasi politiche esistono sempre delle situazioni parziali, nulla si muove in blocco. La società argentina aveva assistito ad una divisione molto profonda

\footnotetext{
${ }^{20}$ Molti pratiche della prima parte del regime militare argentino (1976-1979) ricordano forme e metodi di sistemi politici totalitari o semitotalitari, specialmente con riferimento al concetto arendtiano di atomizzazione della società, connesso all'uso del terrore. Per una prospettiva più ampia Cfr. ARENDT Hannah, Le origini del totalitarismo, Milano, Edizioni di Comunità, Milano, 1967.
} 
del movimento operaio organizzato, fra una burocracia sindacal ovvero un gruppo di dirigenti operai e sindacalisti, che aveva trovato una formula politica nel superamento del sindacalismo come attività di lotta, che si collocava ideologicamente in una destra anticomunista... e fra un sindacalismo di sinistra, che già dall'inizio del Novecento si era fatto strada, che era stato osteggiato dal peronismo nella seconda metà del secolo, in favore di un sindacalismo socialmente avanzato, ma politicamente e ideologicamente inerte. In questo modo si creò una cupula sindical, aldifuori della quale, specie in fabbrica, si costituì un movimento indipendente e di base.

Noi ci inserimmo in questo frangente... dove si costituì un'alleanza fra gli operai della grande industria di Cordoba e Buenos Aires, dalla quale scaturì un nuovo sindacalismo combattente e democratico.

Si costituirono molti movimenti di studenti, intellettuali e operai, che proprio al di fuori del sindacalismo di Stato e della CGT operavano. Per la prima volta nella fabbrica incominciammo a porre la questione politica più che quella salariale e questo ebbe l'effetto, specie in provincia, di scavalcare il sindacalismo ufficiale. Augustìn $\operatorname{Tosco}^{21}$ fu al vertice di questo processo... Un movimento di massa che per primo sarebbe stato represso dai militari. La lezione repressiva che i militari appresero prima del loro arrivo al potere, servì loro per reprimere in maniera straordinaria questo movimento.

\section{R.: I sindacalisti rivoluzionari quindi tennero un collegamento fra intellettuali e operai. In questa fase fu questo il ruolo che svolsero?}

C. N.: Precisamente e, qualche anno prima, (1969) in questo senso il Cordobazo che era nato come movimento studentesco a Cordoba, si unì al movimento operaio e in due settimane l'intera provincia di Cordoba fu presa dal popolo, che rovesciò le istituzioni, anche con l'aiuto della polizia... La repressione di questo momento è uno dei primi passi per i militari nella direzione della repressione totale... gli anni che vanno dal 1967 al 1969 furono di preparazione del golpe, perché si erano resi conto che con una mobilitazione popolare di questa intensità, dove la principale provincia industriale del

\footnotetext{
21 Sindacalista e politico argentino, Tosco fu alla testa del Cordobazo, ma più in generale di un sindacalismo di radice socialista, che molto spesso si avvicinò alle posizioni dei marxisti. Assieme a Raimundo Ongaro fu animatore de la CGT de los Argentinos, fazione di sinistra del sindacalismo ufficiale. Nel 1975 dopo essersi espresso a favore dei rivoluzionari del PRT-ERP passò alla clandestinità. Arrestato e detenuto sotto falso nome dai militari morì lo stesso anno in carcere.
} 
paese rovesciava tutte le sue istituzioni e dove il popolo prende il potere, non poteva che sfociare in una rivoluzione. Se questo fosse successo anche a Buenos Aires allora sarebbe stata una rivoluzione completa.

A partire da qui anche a destra si radicalizzò il conflitto e incominciarono a svilupparsi gruppi paramilitari di stampo fascista, che proprio contro il sindacalismo rivoluzionario si rivolgevano.

\section{R.: In questo quadro che ruolo svolse lei, come decise di diventare militante?}

C. N.: Io appartenevo agli intellettuali, di operaio non ho nulla; andavo all'università, avevo già una professione, però come tanti della mia generazione in questo momento, sentivo la necessità di un cambiamento radicale della società, non si trattava di un mezzo per avere un lavoro, e come tanti, nonostante avessi una vita impostata questa certamente non mi bastava. La militanza appariva come l'esigenza primaria... entrai in contatto con questo mondo che si formava fatto da moltissimo gruppi politici, piccolissimi, ma che stavano vivendo un processo di decantazione e quindi di confluenza all'interno di due o tre tendenze politiche. Un processo che durò cinque anni, fra il 1969 e il 1974, durante il quale i movimenti catalizzarono le energie di tutti. Quando si avviò l'attività armata come risposta alla repressione in atto, prendendo la bandiera della rivoluzione cubana, nell'idea di costituire un fronte latinoamericano, i giovani di questa generazione incominciarono ad andare alcuni nel nord a combattere, altri in Cile con Allende.

Noi eravamo sempre per strada e la gente si attivava politicamente in questo modo. In maniere differenti ci demmo all'attività politica... ma sempre nella strada trovavamo il riscontro e il modo per contattare gli altri...

C. R.: Nel gergo di questi anni la parola che ricorre insistentemente è barrio (quartiere): vivere e far politica nel barrio, pensandolo come ad un luogo dove si incontrava l'altro, che peso ebbe? Oggi le relazioni in un barrio sono differenti; diciamo che sono ridotte, e più che un luogo di incontro è una unità amministrativa/topografica. Negli anni della vostra militanza com'era? 
Bisogna pensare non solo alla struttura sociale, ma anche ai mezzi di comunicazione che negli anni Settanta erano limitati e in questo senso rendevano necessari dei luoghi di riunione sociale. Nei barrios di Buenos Aires le gente partecipava con tutti i problemi che aveva... Tutte le carenze dei servizi, come le scuole, gli asili etc. erano un motivo di incontro e di discussione. La luce, l'acqua e altre utenze muovevano a loro volta tutti... La gente in questo modo parlava e si organizzava... la gente del barrio discuteva non solo il problema contingente, ma si sforzava di comprenderne l'origine, o anche, per quale motivo l'acqua stava in un certo barrio e non in un altro. Da queste riunioni si sviluppò la convinzione che la struttura politica dello Stato e della società andassero rinnovate.... Il nostro lavoro nei barrios fu proprio quello di raccogliere questo sentimento e di convogliarlo nella lotta. Si parlava del barrio e della sua piccola esperienza, ma poi si informavano le persone su quello che accadeva nel mondo e, in questo modo, si diffondeva una certa coscienza. Parlare di quello che accadeva in Cile, in Africa o in Europa coinvolgeva molto la gente del barrio e, successivamente, in molti decidevano di prendere parte alla politica attiva.

\section{R.: Vivere in questa ricchezza sociale del barrio e ad un certo punto ritrovarsi ad avere i militari al suo interno, che effetto vi fece? Fu un trauma?}

C. N: In Argentina, come in tutti i paesi latinoamericani, il rapporto con i militari era differente rispetto all'Europa, ma quando incominciarono ad occupare il barrio, allora fu diverso.

Fra i filmati di guerra della Seconda guerra mondiale dove si vedono i militari nelle strade e l'Argentina del 1976 non c'erano praticamente differenze.

Arrivavano con una unità militare e occupavano... si mettevano nel centro del barrio costituivano un presidio e poi incominciavano a chiudere le strade... successivamente neutralizzavano la politicità del barrio assumendo i vertici dell'opposizione, che magari erano riuniti in sedi politiche e, nel caso di strutture pubbliche, sostituivano i membri della polizia con i militari... e l'amministrazione della sicurezza passava dal civile al militare... ti chiedevano i documenti... controllavano le scuole e l'università... e soprattutto impedivano le riunioni sociali, di qualsiasi origine, e soprattutto quelle politiche. 


\section{R.: E voi continuaste l'attività politica in questo contesto?}

C. N.: L'unica forma che ci era rimasta per riunirci era quella clandestina. Cercando modi differenti di riunione e di discussione politica. Tutto era proibito, non solo la politica attiva, ma anche il teatro, la musica, qualsiasi assembramento sociale. Era una vera e propria occupazione militare. In alcuni luoghi, dove la conflittualità sociale era maggiore, dove si era sviluppata una esperienza di lotta profonda, la repressione passava per una ostentazione di armi... Nella piazza principale di un barrio popolare i militari mettevano un carrarmato e costruivano un presidio con trincee e mitragliatrici, con la presenza fissa di cinque o sei militari... cosa che in un certo senso dichiarava la presenza dei militari non solamente al vertice dello Stato.

Nonostante questo la guerrilla era attiva, e a sua volta per dimostrare di esistere ancora, ingaggiava qualche combattimento per le strade. La guerrilla in Argentina non ebbe mai la forza di ribaltare lo Stato, e quindi si limitò ad azioni di carattere propagandistico, che le servivano per dimostrare a tutti che esisteva ancora. Erano movimenti rapidissimi, piccole azioni ${ }^{22} \ldots$

L'altro grosso problema per organizzare l'azione era la mobilità. Chiunque viaggiasse, sia a Buenos Aires, ma anche uscendo dalla città, era spesso sottoposto a perquisizioni e doveva passare i posti di blocco dei militari. Ti mettevano contro il muro, perquisendoti in cerca di armi, ma soprattutto di libri... Se avevi un bagaglio lo aprivano e ti chiedevano per quale motivo avevi questa cosa o quella... Ovviamente pensare di andare in giro con un libro era una follia... prima te lo avrebbero sequestrato e poi sicuramente saresti scomparso... Noi che eravamo militanti avevamo un doppio problema: chiaramente, già chi non aveva nulla a che fare con la guerriglia rischiava grosso, noi, invece, rischiavamo tutto. Generalmente quasi tutta la struttura militante dei partiti della sinistra dal 1976, l'anno del golpe, fino al 1979, quando la repressione si concluse, fu costretta a passare alla clandestinità perché la repressione era tanto

${ }^{22}$ Cacho Narzole fu militante del principale aggruppamento militare argentino il PRT-ERP (Partido Revolucionario de los Trabajadores - Ejército Revolucionario del Pueblo) che fra il 1973 e il 1976 rappresentò una forza capace di opporre una resistenza ai militari. Nella battaglia di Monte Chingolo (1975) l'esercito rivoluzionario assaltò la caserma dell'esercito argentino con lo scopo di reperire armi. L'operazione non riuscì e si tradusse nella principale battaglia combattuta dalla guerriglia in America Latina. Alla fine del combattimento i militari respinsero l'offensiva durante la quale morirono più di 63 guerriglieri. 
capillare, con una macchina investigativa così ben organizzata, che se venivano nella tua casa o in quella di compagni, e si trovava il tuo nome eri perduto...

Cambiare il nome e ottenere una documentazione falsa poteva essere un modo per eludere questo sistema, se ti avessero fermato per strada, ma ad una investigazione più profonda si sarebbero accorti del trucco.

\section{R.: Che rischio rappresentava per voi lavorare nella propaganda? Come procedevate?}

C. N.: Noi lavoravamo nella struttura di propaganda del partito, che per un movimento è la cosa più importante. Eravamo un canale di comunicazione fra il partito e la gente e il nostro ruolo era difficile perché, non solo dovevamo fare questo, ma anche perché proprio contro la propaganda politica i militari si rivolgevano, perché distruggere la propaganda equivaleva a disinnescare la guerriglia. Noi dovevamo garantire la continuità della comunicazione e dell'informazione per far capire che la resistenza continuava. Senza la propaganda il movimento avrebbe perso totalmente la visibilità... Poi questa si sviluppava in diversi modi: dalla propaganda armata, in questo caso si trattava di una piccola azione dimostrativa in città, alla propaganda informativa, che poteva passare per la pubblicazione di giornali o manifesti. In questo caso noi tagliavamo la strada con il fuoco, ovvero da una parte e dall'altra della strada accendevamo due sbarramenti di fuoco, e all'interno di questo spazio sicuro, tenevamo un discorso pubblico, lanciavamo volantini, e dipingevamo le pareti con scritte contro il regime... tutto questo poteva durare cinque minuti... era una sfida al regime. Il regime in questo modo dimostrava di non essere totale, e la gente non si perdeva del tutto nella paura. L'altra attività di questa propaganda armata era il contatto con la gente, che sostanzialmente si indirizzava alle università o nelle scuole, dove era presente una grande concentrazione di persone, e nelle fabbriche, dove era presente un largo settore di sindacalisti non burocratizzati. Si combatteva con questi metodi, anche diffondendo i giornali clandestini... Quello che invece che fu per noi una sorpresa fu il livello e la violenza della repressione. Noi non pensavamo che con tanta brutalità si sarebbe potuto reprimere il movimento... era un po' l'ingenuità che avevamo che ci faceva pensare questo, un po' anche la mancanza di conoscenza della storia, che è un susseguirsi di 
stragi, ma nonostante questo quando si è dentro la lotta politica ci si sente più forti di quello che realmente si è... La violenza delle forze armate fu di un'intensità tale che superò qualsiasi immaginazione... le torture, i desaparecidos... Noi conoscevamo l'esperienza dei francesi in Algeria, fu una guerra letale, ma la conoscevamo come l'esperienza di un popolo in lotta contro un esercito straniero; in Argentina si propose la stessa situazione, ma in un contesto nel quale non credevamo possibile che ciò avvenisse.

\section{R.: Quanto tempo ci volle per tornare ad una situazione di normalità? Oggi il Ministero della giustizia ha, nella sua denominazione, anche quello di "diritti umani"; nel post dittatura recuperaste subito il terreno perduto ${ }^{23}$ ?}

C. N.: L'intensità della repressione, fu un macigno che si abbatté sulla società argentina: non fu limitata solo agli anni della repressione, ma ebbe ripercussioni per quasi venticinque anni. Nel 1983 subito dopo la caduta del regime, calò il silenzio su quello che era accaduto prima; quando poi, con un grande movimento sociale, arrivò Alfonsín, la società viveva ancora in un profondissimo timore... le violenze furono tali da schiacciare quella vitalità che esisteva prima in Argentina. Sarebbe stato necessario l'arrivo di una generazione, quella del Novanta, per far si che si potesse parlare, ripeto dopo venticinque anni, di queste cose...

La generazione successiva, che non aveva il ricordo della dittatura, incominciò a cercare nuove forme di militanza, dalle quali noi fummo esclusi... Oggi i diritti acquisiti e la libertà di informazione e di movimento, ci portano a interpretare il passato come se si trattasse di un altro mondo. Noi sapevamo questo e quando tornammo eravamo convinti che le esperienze di lotta, che andavano bene per gli anni Settanta, non si sarebbero potute applicare meccanicamente alla nuova situazione politica. Era un altro mondo con

23 Dopo la fine della dittatura militare argentina passarono molti anni prima che i responsabili dei delitti fossero giudicati e prima che si cominciasse a parlare apertamente di quello che era successo durante la dittatura. Solo col governo dei Kirchner il problema della violazione dei diritti umani è stato posto all'ordine del gionro. Simbolicamente la condanna all'ergastolo emessa nei confronti di Videla dello scorso 2011 rappresenta l'impegno dell'amministrazione kirchnerista (peronista di sinistra) di chiudere i conti con la più oscura pagina della storia nazionale. Cfr. COMISIÓN DE EDUCACIÓN DE LA ASEMBLEA PERMANENTE POR LOS DEREECHOS HUMANOS, Memoria y dictadura. Un espacio para la reflexión desde los Derechos Humanos, Buenos Aires, Instituto Espacio para la Memoria, 2008. 
un'altra società e con un altro sistema di valori condivisi. La società evidentemente si è evoluta, oggi siamo sul terreno democratico e riproporre la lotta armata sarebbe una follia. Oggi nessun potere dello stato né democratico, nè de facto, può prevaricare quelli che sono i diritti dell'individuo e anche quelle che sono le reti sociali. Nessuno si sognerebbe di impedire la lotta sindacale, di dire quello che si deve studiare all'università... ci sono altre forme di lotta politica che oggi si possono condurre, ma nella sfera dell'economia mondiale. Dal punto di vista della conformazione della società, gli ultimi cinquanta anni hanno rappresentato una serie di fasi storiche distinte, che ora come stai facendo tu, si possono studiare come qualcosa di finito, di passato... qualcosa di totalmente differente da quello che c'è ora. Io credo però che questo sviluppo sociale che si vede in Argentina e in tutta l'America Latina, oggi, non è il risultato casuale dell'azione politica di noi altri o delle altre generazioni, in quanto si inserisce in un orizzonte di sviluppo storico più generale.

Il lato positivo di questa evoluzione è che molti di qui diritti che rivendicavamo, molti li abbiamo ottenuti per via democratica...

\section{R.: ì quindi questo il coronarsi di un movimento rivoluzionario, nonostante queste vittorie non siano state raggiunte direttamente tramite la vostra lotta?}

C. N.: Semplicemente quello che noi cercavamo di raggiungere con la lotta armata era: un miglioramento di vita della gente, l'estensione dei diritti, le libertà inviolabili, un accesso ai benefici della modernità, l'eliminazione della emarginazione sociale nella quale vivevano le fasce sociali basse... abbiamo ottenuto tutto questo? No, una parte, però chiaramente tutti $\mathrm{i}$ processi politici sono così, nulla si raggiunge mai completamente e poi dobbiamo dire che molte cose che abbiamo ottenuto oggi, prima apparivano come impossibili...

C. R.: In Europa alla conclusione del ciclo di lotte politiche, non seguì un trionfo tale degli ideali di quanti avevano lottato, o non almeno in questa scala, in Argentina sembrerebbe il contrario, volendo paragonare i due casi cosa fece la differenza? 
Io credo che più di Argentina, dobbiamo parlare di America Latina, come una specie di unità strutturale. Così come vi fu in tutto il continente un piano strutturato di dominio di destra $\mathrm{o}$, comunque, se non fu un piano lo fu nella pratica, e fu, in realtà, comune a molti paesi della America Latina... una regola. Noi che abbiamo avuto l'opportunità di stare in Europa e che prendemmo contatti con i movimenti politici italiani di fine anni Settanta... per noi i movimenti politici sembravano sul procinto di conseguire i loro obiettivi e di raggiungere il potere. La sinistra sembrava aver messo in tavola una proposta politica più accattivante di quanto non avesse potuto fare la destra. Ma la successiva evoluzione mondiale della sinistra europea, la proposta politica e valoriale della sinistra si svuotò di significato, confondendosi con la destra. I partiti socialisti di tutta Europa si confonderono a tal punto con la destra, che la loro azione riformista fu praticamente nulla. Le crisi hanno dimostrato proprio questo svuotamento, specie culturale, che ha messo in luce l'assenza di un vero programma politico. Mancò anche un collegamento fra le generazioni che avevano condotto la resistenza e la classe politica che governava nei vari paesi. Questo, diversamente dall'Europa, non accadde in America Latina, dove un nesso generazionale fu stabilito.

Questa assenza di continuità in Europa ancora oggi si sente: i progressisti, in questo modo, ancora oggi si trovano in una condizione di incertezza culturale, come anche in America Latina. Tutto il mondo sta aspettando che in Europa si elaborino nuove idee su come affrontare la crisi, ma soprattutto idee su come costruire una nuova società.

La ripresa dell'America Latina che oggi possiamo notare in larga misura deriva dal fatto che molti settori della sinistra non hanno indugiato quando si è dovuta applicare una politica differente e non hanno rinunciato ai loro valori, che chiaramente hanno adattato al tempo.

Però quello che manca, anche qui, è la rinascita di una intellettualità europea, non chiusa nelle accademie, ma che elabori nuove teorie, non perché manchi un pensiero, ma perché il ruolo degli intellettuali europei è quello di indicare la strada del cambiamento. Il poco che vediamo oggi, sono proposte che ad oggi sono più filosofiche che non politiche.

C. R.: Facciamo un passo indietro; in molti fuggiste in esilio in Europa, in che rapporto vi poneste con i partiti della sinistra, e come viveste questa esperienza? 
Trent'anni fa, quando arrivammo in Italia, era il 1977 e il Pci era il più grande partito comunista e democratico del mondo. Ma nonostante questo noi arrivammo in una Italia dove c'era una avanguardia politica molto avanzata. Quando arrivammo dall'America Latina all'Europa, arrivammo da un mondo dove si erano affermate le dittature militari, ad uno dove la democrazia e il socialismo erano sul punto di trionfare. Il primo impatto con Pci e Psi fu di iniziale diffidenza... convinti di una vittoria democratica delle proprie idee, guardavano a noi, che invece propagandavamo la lotta armata, come se fossimo pazzi. Ma evidentemente noi cercammo di far capire a tutti che esistevano delle differenze tali che non lasciavano lo spazio a soluzioni di tipo politico dei problemi del nostro paese. Le strutture di dominazione, di dipendenza feudale in campagna e di stringente lobbismo che erano parte della struttura sociale argentina, chiaramente imponevano una scelta drastica.

In più la caduta di Allende in Cile testimoniava come una soluzione di stampo europeo, ovvero di affermazione politica socialista e non violenta, non poteva funzionare in un contesto politico marcato dalla violenza politica.

Stemmo due anni in Italia e, in questo tempo, mettemmo su una scuola politica, con il sostegno dei movimenti politici dell'avanguardia. Tutta quella galassia di partiti extraparlamentari, critica verso i partiti della sinistra parlamentare, ci dette aiuto. Parlo di Democrazia proletaria e altri, anche se noi ci trovammo in un momento in cui questi partiti erano cresciuti molto, ma avevano raggiunto un livello oltre il quale non potevano andare. La guerriglia era uno dei temi di cui si parlava, e con la storia della RAF in Germania la discussione si era radicalizzata ulteriormente... Gli extraparlamentari furono inizialmente i più vicini alle nostre posizioni, e furono quelli che ci dettero un aiuto iniziale. Successivamente però prendemmo contatti con il Psi e il Pci del Piemonte. Ai partiti si aggiunse anche la CGIL, che ci aiutò in conferenze e incontri a spiegare al pubblico europeo quello che accadeva in Argentina. Noi dovevamo rompere un silenzio che esisteva in Europa sull'Argentina, che era anche fomentato dalla mancanza di libertà di stampa.

Con l'occasione della fuga dall'Argentina riemersero quei legami fra Italia e Argentina, legami di emigrazione, che ci aiutarono molto. Quasi tutti quelli che vennero con me avevano qualche parente in Italia. Questo ci garantì una grande appoggio. Noi stemmo 
due anni in Italia, ma nel frattempo in Argentina ci furono grossi cambiamenti: la stessa proposta politica della giunta militare, sembrò più accettabile di quanto non lo fosse stata prima.

In questa fase la massa di morti che i militari aveva creato incominciava a pesare sulla società argentina e, in secondo luogo, a mettere in crisi - con dispute per il potere e tutto il resto - i militari. Il costo politico dell'uccisione sistematica di trentamila persone non poteva non giocare un ruolo in una fase discendente della giunta... questa era qualcosa che non potevano più nascondere, e che con il passare del tempo saltava alla luce del sole. In questo modo si creò un rifiuto verso la giunta militare, che poi si fece concreto quando l'Argentina si imbarcò nella follia delle Malvinas... con la guerra con l'Inghilterra... con tutto quello che seguì. Ma ripeto anche questa evoluzione rispondeva ad una logica globale di sviluppo dei regimi politici della America Latina.

\section{R.: Questa è la risposta che mi aspettavo da un militante rispetto alla fuoriuscita; ma come uomo, come visse fuori dall'Argentina in questi anni?}

C. N.: Le esperienze sono state molto diverse... ovviamente non tutti abbiamo avuto la stessa sensibilità per comprendere quello che accadeva. Il nostro gruppo stette in Italia tre anni e altri due in Messico. Io personalmente non considerai un esilio questo tempo... Quando uscimmo dall'Argentina, scappammo in gruppo, e quindi non scappai come singolo che stava per essere preso dall'esercito. Chiaro che se mi avessero perquisito avrei fatto quella fine... Uscimmo fuori dall'Argentina come struttura politica coerente, con il chiaro obiettivo di riorganizzare la lotta dall'Europa e ritornare per poter vincere. Questo era il piano originario, ma poi l'evoluzione dei fatti avrebbe fatto si che non lo rispettassimo... ma questo accade sempre... In Argentina la società cambiava con una velocità tale, che da fuori non potevamo comprendere...

Per me l'esperienza dell'esilio fu di grande accrescimento: umanamente ha contribuito alla mia formazione come uomo, prima che come militante. Anzitutto perché in Italia ${ }^{24}$

\footnotetext{
${ }^{24}$ Cacho fu ospitato con la famiglia nel paese di Naviante (CN) dove grazie ai rapporti con la locale sede della CGL potè lavorare e vivere due anni. Qualche anno dopo lo stesso protagonista di questa intervista scrisse Tributo a Naviante, libro di memorie sull'esperienza dell'esilio. Un articolo di Papuzzi uscito su «La Stampa» ricostruisce questa esperienza. L'articolo è consultabile all'indirizzo, URL:

< http://www1.lastampa.it/redazione/cmsSezioni/cultura/200711articoli/27901 girata.asp > [consultato il 10 gennaio 2013]
} 
conobbi persone con le quali ho condiviso tanto, che ancora oggi sento. Si è costruita una rete affettiva talmente grande, che chiaramente ha una origine e una motivazione politica, ma che è andata oltre.

Questo rapporto di fraternità si sviluppò in maniera reciproca per un motivo preciso: le forme erano distinte, ma la necessità di partecipazione politica era quella che univa la nostra come tutte le generazioni... Volevamo una società migliore, niente di più. I rapporti umani furono agevolati da questa istanza comune, il desiderio di un mondo migliore e di lottare per raggiungerlo. Le esperienze furono diverse, ma l'orizzonte era comune.

Per me questo periodo in Italia fu qualcosa di inimmaginabile: non solo si instaurarono amicizie, ma per esempio fui invitato al congresso di Novara della CGIL, dove i delegati sindacali e politici mi fecero sedere al centro del tavolo della conferenza. Parlare ad una enorme platea della condizione dell'Argentina fu una esperienza di affetto indimenticabile... Mi pareva di parlare all'avanguardia operaia del mondo, questo mi fece crescere molto come persona.

Quello che fu umanamente la mia vicenda, fu anche una storia di quotidianità, di risoluzione dei problemi che si ponevano per mandare le mie figlie a scuola, per trovare una casa ed un lavoro.

L'umanità dei compagni emerse anche in questo momento, quando ottenemmo che le mie figlie sarebbero potute andare all'asilo senza alcun documento, che ovviamente non avevamo. In questo frangente le maestre si organizzarono con la direzione della scuola per farle stare lì. Allo stesso tempo in ospedale ottenemmo lo stesso trattamento.

Una profonda concezione che mi ha lasciato questo periodo è proprio quella che il mondo non è uno schifo come spesso possiamo immaginare, ma anzi questa esperienza ci dimostrò l'opposto. È chiaro che non tutti vivemmo in questo modo l'esilio, ma per me era così.

Una esigenza che sentivo era quella di lavorare. Io ero professionalizzato e laureato, ma trovai lavoro come imbianchino. Il sindaco di Montalto Dora, che era mio amico, mi aiutò a trovare questo lavoro. Un giorno mi disse «devi dipingere tutte le ringhiere dei ponti che ci sono» ed io «perfetto» ovviamente non avevo documenti, ma lavorai senza problemi, e tutti nel paese che conoscevano la nostra situazione ci aiutavano come potevano. 
Alcuni compagni soffrirono molto questa lontananza, ma io penso ancora a questo momento come ad uno dei più importanti della mia vita. Mi figlia stessa ricorda questa esperienza molto bene, è tornata varie volte in Italia, dove ha molti amici fra i figli di quanti ci aiutarono in Piemonte. Oggi un mio vecchio amico di partito mi ha rivelato che adesso quello che accadde con noi non sarebbe più possibile... Per me questo non ha un valore decisamente negativo o positivo, non dobbiamo giudicare con meglio e peggio quello che è, siamo davanti e stiamo parlando di un altro mondo, con altre relazioni sociali e con altri problemi. Nonostante questo alcuni rapporti possono sopravvivere a questa evoluzione.

Le società si modificano, e con esse anche i suoi rapporti, quando arrivammo in Piemonte in questo paesino di contadini, non c'era nulla di quella tecnologia che oggi ha impresso una rivoluzione totale alla comunicazione e ai rapporti umani.

\section{R.: Parliamo del suo ritorno in Argentina, come lo visse?}

C. N.: Il ritorno fu un'esperienza molto complicata, fu personalmente molto duro. La violenza psicologica che gli anni della giunta militare impressero al paese si sentiva molto. L'idea che avevamo era che, appena tornati, anche per l'esperienza di crescita politica maturata in Europa, saremmo subito tornati all'attività politica. Arrivammo alla fine del 1983 in Argentina, poco prima della elezione di Alfonsín. Vivemmo giorni di grande euforia politica, se pur non con l'intensità di quelle manifestazioni politiche del 1976. Il timore che aleggiava nella società era la grande novità che trovammo, e, nonostante la partecipazione politica fu forte, nessuno intendeva compromettersi. L'individualismo si era installato in una misura che mai si era vista prima. Era evidente che l'azione psicologica brutale della dittatura aveva contribuito a questa evoluzione. Ma non era solo questo: non esistevano più studenti politicizzati nelle università, non esistevano più formazioni sindacali che non fossero quelle burocratizzate come la CGT, mancavano centri sociali o luoghi dove l'arte e la cultura si potessero esprimere liberamente. Nulla di quella cultura dell'Argentina pre dittatura era rimasto in piedi.

In questo nuovo contesto tentammo di avviare una attività politica con alcuni settori della politica ufficiale, ovviamente progressisti, però senza uscire dalla logica dei partiti 
politici argentini, che avevano agevolato l'uscita morbida dei militari e l'impunità, in cambio di un ritorno alla democrazia.

Per far questo i partiti politici maggioritari ci imposero un blocco. Fu molto difficile far politica in un contesto dove i partiti politici avevano occupato tutto lo spazio. L'alfonsinismo segnò un periodo dove si utilizzavano molto le parole, ma dove queste avevano poco o nessun significato.

\section{R.: Il governo di Menem si inserì quindi in questa scia?}

C. N.: Non precisamente: il menemismo dimostrò che si poteva gestire il potere, come lo aveva fatto la giunta militare, ma senza dittatura.

Evidentemente queste figure si inseriscono in un momento, che è quello del neoliberalismo, che globalmente ebbe le proprie manifestazioni. Gli anni di Reagan e la Thatcher si immettono pienamente in questo momento.

In questo quadro noi vivemmo difficoltà per far politica, ma anche per rientrare nel mondo del lavoro. Le difficoltà del ritornare a far politica erano rappresentate dalla difficoltà di scegliere come portare avanti qualsiasi azione. La via democratica appariva serrata ed alcuni gruppi più radicali tornarono a modelli di lotta armata, come quelli che avevamo sperimentato negli anni Settanta. Ma in quest'ultimo caso la clandestinità di un piccolo partito rivoluzionario, che continuava a pubblicare manifesti e libri con le stesse parole di prima, «revolucíon», «socialismo», «clase obrera» appariva come totalmente staccato dalla realtà del paese.

Noi capimmo che questo modo di combattere non poteva funzionare più $\mathrm{e}$ appoggiammo la parte più progressista dei partiti per dare continuità alla nostra lotta.

Era ovvio che i metodi che utilizzammo furono quelli di una nuova generazione, che con la guerriglia e l'azione rivoluzionaria non aveva nulla a che fare.

Ognuno può trarre le proprie conclusioni, ma era chiaro che l'azione politica degli anni Settanta non sarebbe potuta essere uguale a quella degli anni Ottanta e Novanta.

Ammettere questo cambiamento anche generazionale era un dovere anche verso noi stessi, eravamo quasi sui cinquant'anni e dovevamo ammettere che eravamo fuori.

In questo senso tentammo di porre un ponte fra noi che continuavamo con una militanza che non sapevamo bene dove collocare e i giovani di vent'anni che si affacciavano alla 
politica attiva. Conoscere questi nuovi militanti ci fece carpire che non c'era la possibilità di una continuità nei metodi e nelle lotte fra noi e loro, perché il loro era un interesse e un panorama diverso... il loro interesse nei nostri confronti era un'altra cosa...

\section{R.: Possiamo dire che era un interesse storico, o di memoria, più che programmatico?}

C. N.: Sì! Era esattamente la necessità di ricostruire un periodo che appariva come finito. E parlammo. Si andava nelle fabbriche a parlare di questa esperienza. Il lato pedagogico prevaleva su quello politico. Andammo in fabbriche che erano occupate dagli operai che aderivano al movimento - parliamo degli anni Novanta - fabrica recuperda che in alcuni casi espropriarono le fabbriche stesse collettivizzandole.

Ci chiesero, i comitati di fabbrica, come organizzarle... anche perché avevamo fatto alcune esperienze del genere prima del golpe, ma noi davanti ad alfonsinismo e menemismo non sapevamo che strada trovare, come organizzare socialmente tutto questo.

Dopo anche nell'università ci si cominciò ad interessarsi alla nostra esperienza politica, chiaramente con un interesse storico. Poi incominciarono a scriversi libri e girarsi film su questa esperienza della dittatura, che finalmente usciva dall'anonimato in cui era rimasta celata. L'arrivo del Kirchnerismo ${ }^{25}$ (2003) con tutto l'apparato di riscoperta dei diritti umani, è stato per noi importantissimo.

Molti ex militanti dei partiti rivoluzionari come noi altri, noi della generazione del Settanta, entrammo in questo processo.

\section{R.: Possiamo intravedere un collegamento fra la vostra generazione e il}

\section{Kirchnerismo?}

\footnotetext{
25 Legato alla figura del presidente Nestor Kirchner, che dopo la crisi economica del 2001 guidò l'Argentina in una fase di ripresa economica e di sviluppo della piena occupazione. Il Kirchnerismo si configura come un'azione di governo classicamente peronista, ma con un marcato accento, soprattutto simbolico, di sinistra. Il Kirchnerismo in questo senso oltre a colpire i simboli dell'ultima dittatura militare, e a riaprire i processi a carico dei militari coinvolti nella faccenda dei desaparecidos, (amnistiati da Menem) integra tutt'ora nel governo di Cristina Fernandez de Kirchner (moglie di Nestor tutt'ora presidente in carica al secondo mandato dell'Argentina) molte personalità legate ai movimenti rivoluzionari di sinistra (PRT-ERP) e peronisti (Montoneros) che furono perseguitati durante l'ultima dittatura militare.
} 
Certamente il Kirchnerismo ha prodotto questa connessione fra l'azione politica che avevamo portato avanti negli anni Settanta e quella attuale. Non è un caso che proprio i governi di questi anni hanno generato una reazione fortissima di destra. Una destra che oggi non può sopportare, un'altra volta, che torni a galla qualcosa che erano convinti di aver distrutto, con tanta determinazione e con tanto sangue... e che nonostante tutto torna. Torna attraverso altre forme, ma nella sostanza la stessa gente che nel Settanta era impegnata nella lotta armata, oggi democraticamente sta raggiungendo gli stessi obiettivi che aveva trent'anni prima.

\section{R.: In questo senso la forma della lotta che ruolo ha svolto in una più larga prospettiva?}

C. N.: Le forme politiche e di lotta, sono secondarie, si adattano al momento storico: quello che abbiamo sempre ritenuto importante è l'obiettivo. Il nostro gruppo decise proprio di abbandonare la lotta armata: l'obiettivo finale era il miglioramento delle condizioni delle classi basse, e non la lotta in sé.

Credo che l'esperienza dell'Italia ci favorì in questa scelta perché noi altri prendemmo in quei due anni molti contatti con i partigiani, che erano vecchi partigiani. Ascoltavamo con grande emozione $\mathrm{i}$ racconti dei partigiani e proprio da questo incontro comprendemmo che lotta armata e lotta politica, potevano essere utilizzate in modo alternato e non l'una in opposizione all'altra.

$\mathrm{Fu}$ per questo che accettammo di supportare questi settori, che hanno compiuto grossi passi avanti nella direzione dello sviluppo democratico.

Alcuni di noi hanno mantenuto la loro linea politica, ovviamente senza la lotta armata, continuando con un complottismo parallelo, fuori dal tempo. Noi che oggi abbiamo sessant'anni come potevamo continuare in questa direzione?

Tutta questa nostra conversazione ha avuto un valore di riepilogo di quella che è stata la storia argentina degli ultimi quarant'anni, ma spero abbia messo in luce un altro elemento, quello centrale, ovvero la partecipazione di massa di un'intera generazione argentina alla militanza politica, in tutte le sue forme. Una partecipazione molto spesso disinteressata il cui fine era cambiare il mondo. 
C. R.: Mi sembra però che da questa nostra conversazione emerga anche un altro elemento; la sostanziale unità di intenti, di riferimenti culturali e politici, non solo di una generazione argentina, ma di tutte le generazioni degli anni Settanta.

C. N.: Effettivamente l'idea di cambiare il mondo non era solo nostra, ma apparteneva a tutti i giovani. La lotta contro la guerra del Vietnam effettivamente dimostrò quello che hai detto. La partecipazione mondiale di intellettuali giovani e artisti è un fenomeno particolare, che non è affatto comune. Questo fu un momento particolarmente... credo che gli anni Settanta furono e rappresentarono un momento di cambiamento, talmente profondo, da ribaltare un intero assetto sociale. Prima le élites sociali avevano mantenuto sotto il loro potere intere classi, che proprio in questi anni, irruppero nella vita politica come mai prima. La gente per le strade modificò la società... nulla sarebbe potuto più essere come prima. 


\section{* L'autore}

Camillo Robertini si è laureato in storia a Perugia e Venezia; ha svolto un periodo di studio in Argentina (doble titulación) durante il quale ha realizzato un primo programma di interviste a militanti rivoluzionari e sindacali. Ha presentato i primi risultati delle proprie ricerche al $\mathrm{XXXV}^{\circ}$ Convegno internazionale di americanistica di Perugia (maggio 2013). Collabora con la rivista argentina «Prohistoria» e recentemente ha curato la pubblicazione del volume Questa terra è la mia terra, storie dal Veneto dal Salento e dall'America Latina (Latina, La Toletta, 2013).

URL: < http://www.studistorici.com/progett/autori/\#Robertini >

\section{Per citare questo articolo:}

ROBERTINI, Camillo, «"A vencer o morir": rivoluzione e militanza politica nell'Argentina degli anni Settanta. La storia di Cacho Narzole rivoluzionario ed esule politico argentino», Diacronie. Studi di Storia Contemporanea : Spazi, percorsi e memorie, 29/10/2013,

URL:< http://www.studistorici.com/2013/10/29/robertini_numero_15/ >

Diacronie Studi di Storia Contemporanea $\beta$ www.diacronie.it

Risorsa digitale indipendente a carattere storiografico. Uscita trimestrale. redazione.diacronie@hotmail.it

Comitato di redazione: Marco Abram - Jacopo Bassi - Luca Bufarale - Alessandro Cattunar - Elisa Grandi - Deborah Paci - Fausto Pietrancosta - Matteo Tomasoni - Luca Zuccolo

Diritti: gli articoli di Diacronie. Studi di Storia Contemporanea sono pubblicati sotto licenza Creative Commons 2.5 Possono essere riprodotti a patto di non modificarne i contenuti e di non usarli per fini commerciali. La citazione di estratti è comunque sempre autorizzata, nei limiti previsti dalla legge. 